\title{
III-V-on-silicon mode-locked lasers with 1-GHz line spacing for dual-comb spectroscopy
}

\author{
K. Van Gasse, ${ }^{1}$ J. Huh, ${ }^{2}$ Z. Chen, ${ }^{2}$ S. Poelman, ${ }^{1}$ Z. Wang, ${ }^{1}$ G. Roelkens, ${ }^{1}$ T.W. Hänsch, ${ }^{2,3}$ B. Kuyken, ${ }^{1}$ \\ N. Picqué ${ }^{2}$

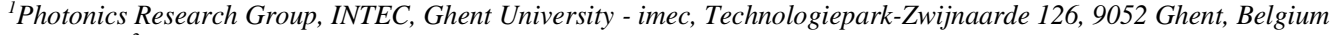 \\ ${ }^{2}$ Max-Planck-Institut für Quantenoptik, Hans-Kopfermann-Straße 1, 85748, Garching, Germany \\ ${ }^{3}$ Ludwig-Maximilians-Universität München, Fakultät für Physik, Schellingstr. 4/III, 80799, München, Germany \\ kasper.vangasse@ugent.be
}

\begin{abstract}
We demonstrate dual-comb interferometry and spectroscopy with a III-V-on-silicon passively mode-locked laser of $1-\mathrm{GHz}$ repetition rate and $1-\mathrm{THz}$ span. We heterodyne the on-chip device with an electro-optic modulator comb for initial assessment.
\end{abstract}

\section{Introduction}

The development of chip-scale frequency combs is an active field of research [1]. Several solutions are currently explored, involving Kerr combs, electro-optic (EO) combs or quantum and interband cascade lasers. However, almost all demonstrated systems involve large line spacing, of tens of $\mathrm{GHz}$ or even more. Many applications would benefit from chip-scale frequency combs with a smaller line spacing $(\leq 1 \mathrm{GHz})$. One of these is molecular spectroscopy and sensing in the gas phase, where the transitions at atmospheric pressure have a width of the order of a GHz. Therefore, comb line spacings of $\leq 1 \mathrm{GHz}$ are needed to properly sample the spectra without interleaving.

Here we explore the potential of III-V-on-silicon mode-locked lasers with a 1-GHz line spacing [2], for dual-comb spectroscopy [3]. Dual-comb spectroscopy is a technique of Fourier transform spectroscopy without the need for moving parts. Using this technique a broad spectral span can be interrogated in a short time with an unparalleled consistency of the spectral data. Two combs of slightly different repetition frequencies are heterodyned. The beat notes between pairs of optical comb lines, one from each comb, produce a radio-frequency (RF) comb that can be efficiently processed by digital electronics. The generation of the RF comb with narrow comb lines is necessary for high-resolution and high-sensitivity measurements, although it is challenging to achieve because two low-noise frequency combs are needed. This puts very strong constraints on the noise properties of the comb lasers.

Photonic-chip-based semiconductor mode-locked lasers are very appealing sources for dual-comb spectroscopy because of their compact formfactor and mature fabrication technology. However, so far they have not been useful for dual-comb spectroscopy because of their wide optical linewidth (>10 MHz) and noise properties. III-V-onsilicon technology allows us to realize an on-chip mode-locked laser with beyond state-of-the-art performance, because of the combination of high-performance III-V gain material with low-loss silicon waveguides. The low-loss silicon waveguides enable the creation of a long on-chip extended cavity, providing the laser with both narrow optical linewidth and a low repetition rate [2]. Both of these qualities are needed for dual-comb spectroscopy of molecules in the gas phase. Furthermore, such a III-V-on-silicon laser can be fabricated on a wafer-scale by leveraging mature CMOS fabrication techniques.

We demonstrate for the first time that an on-chip mode-locked laser, shown in Fig. 1(a), can be used for dual-comb interferometry and present the experimental results of heterodyning the chip-comb with an EO comb for initial assessment.

(a)

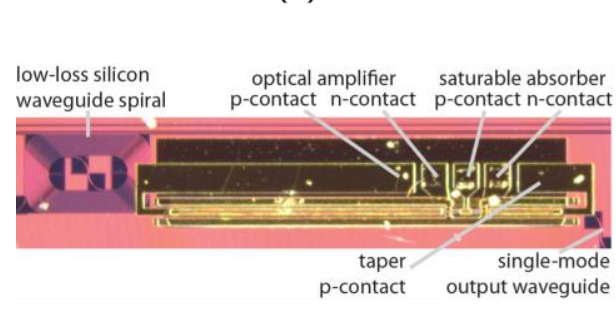

(b)

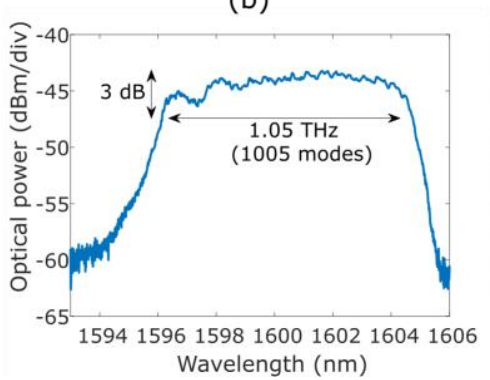

(c)

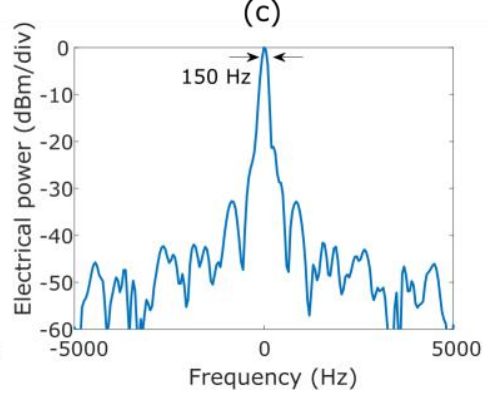

Figure 1: (a) Microscope image of a III-V-on-silicon mode-locked laser with the key-elements annotated. (b) Optical spectrum (50 pm resolution) of the III-V-on-silicon mode-locked laser comb with $1 \mathrm{GHz}$ linespacing. Individual comb lines could not be resolved by the OSA. (c) Electrical spectrum centered on the repetition rate signal generated by the III-V-on-silicon mode-locked laser (10kHz span, $150 \mathrm{~Hz}$ RBW, $150 \mathrm{~Hz}$ VBW). 


\section{Experimental results}

The III-V-on-silicon mode-locked laser chip was placed on a temperature-controlled chuck and it was electrically contacted using a custom-designed RF probe, which allows biasing the saturable absorber and the gain sections separately. The optical output of the laser is collected using a cleaved standard single-mode fiber and a chip-to-fiber grating coupler. The $1.05 \mathrm{THz}$-wide optical spectrum is measured using a $50 \mathrm{pm}$ resolution optical spectrum analyzer and shown in Fig. 1(b). The repetition rate of the passively mode-locked laser is determined by sending the optical output to a photoreceiver with a trans-impedance amplifier and analyzing the output with an Electrical Spectrum Analyzer (ESA). The repetition rate is found to be $1009730 \mathrm{kHz}$ and shows a drift of about $\pm 3 \mathrm{kHz}$ on a $100 \mathrm{~ms}$ time-scale. A spectrum $(10 \mathrm{kHz}$ span) centered on the repetition rate is shown in Fig. 1(c). For III-V semiconductor mode-locked lasers, the stability of the repetition rate is often expressed as the $3 \mathrm{~dB}$ RF linewidth of the repetition rate signal. For this laser, the repetition rate signal width at $3 \mathrm{~dB}$ is $150 \mathrm{~Hz}$, which is the narrowest reported so far for a semiconductor mode-locked laser to the best of our knowledge. This beyond state-of-the-art performance, together with the narrow optical linewidth $(<1 \mathrm{MHz})$, makes it possible, for the first time, to use an integrated mode-locked laser for dual-comb interferometry and spectroscopy.

To assess the dual-comb capabilities of the laser, we constructed an EO comb with a repetition rate of $1000 \mathrm{MHz}$ and we optically combined it with the output of the III-V-on-silicon mode-locked laser, as shown in Fig. 2(a). This combined output is then sent to a balanced photodetector (Thorlabs PDB481C-AC) to generate the dual-comb interferogram. To preserve the linearity of the photodetector, the average optical power incident on the photodetector is approximately $100 \mu \mathrm{W}$. The output of the balanced detector is recorded with a data acquisition card (ATS9360-12 bit, $1.8 \mathrm{GS} / \mathrm{s}$ ). The recorded time trace (of $1 \mathrm{~ms}$ ) is then divided into slices and a Fourier-transform is used to generate the dual-comb spectrum shown in Fig 2(b). The beat notes are spaced by $9.73 \mathrm{MHz}$ and have a fullwidth at half maximum of approximately $1 \mathrm{MHz}$. These results clearly illustrate the unique performance of our laser, as most integrated mode-locked lasers have an optical linewidth exceeding $10 \mathrm{MHz}$, which would make it impossible to generate a dual-comb spectrum.

(a)

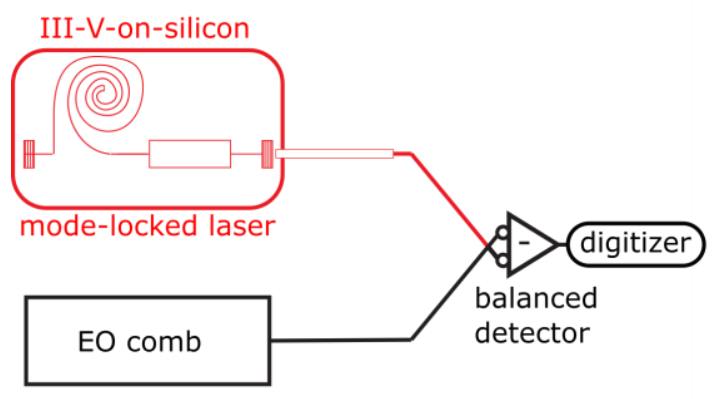

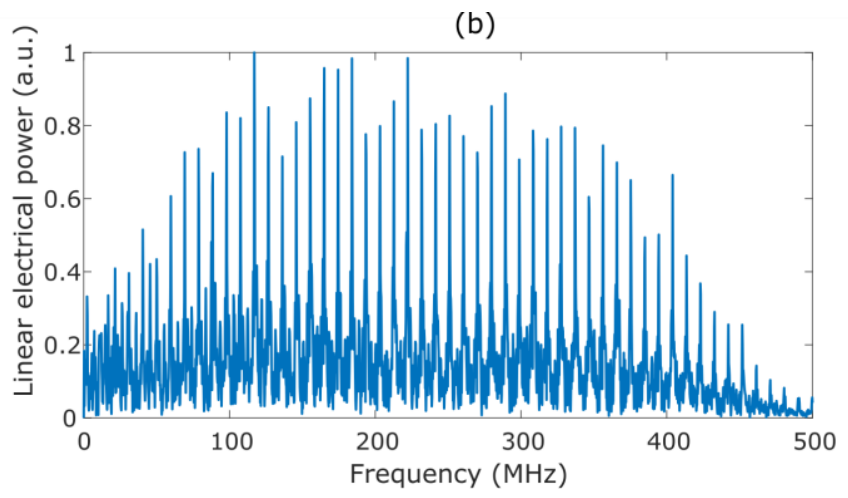

Figure 2: (a) An overview of the measurement set-up use to generate the dual-comb set-up. (b) The spectrum generated by taking the Fouriertransform of 1 us slice of the dual-comb interferogram.

\section{Conclusion and outlook}

The presented results pave the way for dual-comb spectroscopy using III-V-on-silicon mode-locked lasers. Furthermore, these devices can be fabricated on a wafer-scale and unlike Kerr or electro-optic combs do not require an off-chip light source. Given that long low-loss waveguides for sensing and photodetectors could be integrated on the same chip as these III-V-on-silicon mode-locked lasers, a fully integrated dual-comb spectrometer could be realized in the future.

\section{References}

[1] A. L. Gaeta, M. Lipson, T. J. Kippenberg, "Photonic-chip-based frequency combs," Nat. Photonics 13, 158-169 (2019).

[2] Z. Wang, K. Van Gasse, V. Moskalenko, S. Latkowski, E. Bente, B. Kuyken, G. Roelkens, “A III-V-on-Si ultra-dense comb laser,” Light Sci. Appl. 6, e16260 (2017).

[3] N. Picqué, T. W. Hänsch, “Frequency comb spectroscopy,” Nat. Photonics 13, 146-157 (2019). 